\title{
Decisiones compartidas en la enfermedad avanzada
}

\author{
Shared decision-making in advanced disease
}

\author{
Mª Aránzazu García Martínez ${ }^{1,3}$, Isabel Vila Iglesias ${ }^{2,3}$, Dolores Veiga Cando ${ }^{2}$ \\ ${ }^{1}$ Médico de la Unidad de Hospitalización a Domicilio. Hospital Universitario Lucus Augusti. SERGAS. Lugo \\ 2Enfermera de la Unidad de Hospitalización a Domicilio. Hospital Universitario Lucus Augusti. SERGAS. Lugo \\ ${ }^{3}$ Miembro del Comité de Ética Asistencial. Área sanitaria Lugo centro
}

Tomar decisiones es una práctica diaria de todo ser humano. Hay decisiones sencillas, con pocos valores en juego, con consecuencias poco importantes y en las que solo una persona está implicada y otras más complejas y con consecuencias irreversibles.

En los escenarios cercanos al final de la vida hay dos momentos especialmente difíciles en cuanto a la toma de decisiones ${ }^{1}$ : el momento de la transición al cuidado paliativo y el muy cercano al final en que el profesional se plantea una Adecuación terapéutica / Limitación del esfuerzo terapéutico / Sedación, etc. En ambos hay un cambio en la actitud terapéutica: renunciamos al curar y ponemos el "cuidar" como objetivo fundamental. Aceptamos los límites de la medicina por un lado y la finitud de la vida por otro.

Dejaremos a un lado los escenarios finales y nos centraremos en la toma de decisiones desde el momento en que a un enfermo se le diagnostica una enfermedad incurable, en que se concluye que ya no existen tratamientos curativos y los objetivos terapéuticos serán exclusivamente paliativos.

\section{Caso clínico}

Manuel es un paciente de 45 años, casado y con dos hijos, licenciado superior. Fue remitido por primera vez a nuestra unidad para apoyo global domiciliario tras colocación de traqueostomía conectada a ventilación mecánica invasiva (VMI) y sonda de gastrostomía para alimentación.

Había sido diagnosticado dos años antes de Esclerosis lateral amiotrófica (ELA) en el Servicio de Neurología. El propio paciente buscó información cuando comenzaron sus síntomas de debilidad, caídas frecuentes, etc., y cuando acudió a la primera consulta del neurólogo, le dijo que creía que tenía ELA. Desde el diagnóstico el paciente había pedido una segunda opinión a un neurólogo de otra ciudad, quién solicitó su consentimiento para ser incluido en un ensayo clínico.

Conocedor de su diagnóstico y pronóstico, fue portador de ventilación mecánica no invasiva durante varios meses. Aficionado a la poesía, había comenzado a escribir en los últimos tiempos, actividad con la que afirmaba disfrutar muchísimo. Vital y optimista por naturaleza, disfrutaba de la relación con su familia, amigos, y de varios niños que habían nacido en la familia en los últimos meses.

Ante un empeoramiento evidente y progresivo de su enfermedad, su neumólogo le planteó la colocación de VMI. Se informó acerca de sus posibilidades, de su vida futura conectado a un respirador, y su respuesta fue afirmativa: "adelante, aún me quedan muchas cosas por hacer". Tras casi dos meses en el Hospital y numerosas complicaciones, se consideró que estaban preparados para trasladarse a su domicilio. Se les ofreció apoyo del servicio de Hospitalización a domicilio (HADO), que aceptaron. Contactaron antes del alta con una cuidadora que ayudaría a su mujer.
Conocimos al paciente en su domicilio; se comunicaba a través de un panel con letras y números. Manejaba perfectamente el ordenador, herramienta de trabajo habitual, con el que escribía poesía y cuentos. Conservaba movilidad escasa en la extremidad superior derecha y ninguna en la izquierda e inferiores. Emitía sonidos. Se mostraba contento, aceptando la situación.

Durante 1 ó 2 meses realizamos visitas frecuentes en su domicilio. Además del adiestramiento en el uso de la sonda de gastrostomía y de todo lo relacionado con la ventilación mecánica "hablábamos" de la vida, de su vida, de su lucha contra la enfermedad, de sus valores, que casi sin quererlo iba revelando. Siempre manifestó que para él la vida tendría sentido mientras tuviera algún modo de comunicarse, de interaccionar; el día en que eso no fuera posible no desearía seguir viviendo.

Se realizaron dos visitas conjuntas con el equipo de atención primaria y se consiguió que su enfermera asumiera los cambios de cánula de traqueostomía.

\section{Fundamentación}

La expresión Toma de Decisiones Compartidas (TDC) (Shared Decision-Making en la literatura inglesa) aparece por primera vez en 1982 en EEUU y fue definida como "un proceso de mutuo respeto y colaboración para la toma de decisiones en materia sanitaria", por la President's Comission for the Study of Ethical Problems in Medicine and Biomedical and Behavioral Research, en su informe Making Health Care Decissions, centrado en el Consentimiento Informado?

Tras más de 30 años intentado trasladar esta idea a todas las decisiones clínicas y con numerosas aproximaciones y definiciones en la literatura, actualmente se puede definir la TDC como la interacción médico-paciente en la que hay dos vías de intercambio de información con la participación de ambas partes en la decisión ${ }^{3}$. También como un proceso de decisión conjunta entre pacientes y profesionales sanitarios, que tiene como principal objetivo que los pacientes estén informados y adopten un papel más activo en las decisiones sobre su salud". Y "estilo de relación mediante o cal o profesional aplica coñecementos científicos e habilidades de comunicación tendo en conta os valores e preferencias do paciente para axudarlle e apoialo na súa decisión" 5 .

Se ha observado que cuando los pacientes son informados de una manera más apropiada sobre las diferentes opciones terapéuticas, pueden alcanzar decisiones de mejor calidad6. Tal es la evidencia científica que incluso desde los Servicios Públicos de Salud tanto internacionales ${ }^{7}$ como autonómicos $^{4}$ (Andalucía, Canarias, Madrid) se han desarrollado herramientas para la toma de decisiones compartidas. En Galicia, con 
el objetivo de "servirlles de marco de referencia e orientación aos profesionais e usuarios sobre os conflitos éticos que xorden nesta fase da vida, e contribuír a mellorar o desenvolvemento dos dereitos fundamentais neste ámbito", se ha publicado un documento de recomendaciones ${ }^{5}$ referidas fundamentalmente a procurar "una xestión máis autónoma do proceso do final da vida" (dicho documento ha sido recientemente objeto de un artículo de revisión en esta revista ${ }^{8}$ ).

En el final de la vida, en cualquier fase desde que se diagnostica una enfermedad avanzada y terminal, las situaciones de incertidumbre aumentan, y no están claras las posibles ventajas de unas opciones clínicas sobre otras. La participación del paciente en la toma de decisiones en esta fase de la vida es de tal importancia que para el profesional se convierte en un imperativo moral. Algunos autores ${ }^{1}$ proponen un modelo de toma de decisiones basado en el modelo de relación clínica deliberativo ${ }^{9}$. El profesional tiene el deber de transmitir la información y las opciones de una manera clara, veraz y comprensible, de explorar los valores del paciente y según ellos sus preferencias; ambos entrarán en un proceso de deliberación en el que también estarán en juego los valores del profesional. En este modelo de TDC hay varios elementos claves:

- El proceso de transmisión de la información y la recepción de la misma por parte del paciente.

- La consideración de la autonomía del paciente como un valor fundamental, por encima del principio de beneficencia en que se basa el modelo de relación médico-paciente paternalista (modelo más clásico).

- El proceso de deliberación en sí de cara a tomar la mejor decisión, la más prudente.

- El Acompañamiento por parte del profesional implicado, lejos también del modelo de relación médico-paciente informativo en el cual el profesional es un mero transmisor de información veraz sin otra implicación que la necesaria para la realización de su trabajo.

Analizaremos brevemente cada uno de los apartados anteriores.

\section{Transmisión de la información}

El acceso a la verdad es un derecho del paciente. Pero ¿cuál es la verdad que debemos transmitir? ¿Es maleficente decirle a alguien que su enfermedad ya no tiene posibilidades de curación? ¿0 el hecho de conocer esta verdad le puede ayudar a tomar determinadas decisiones, a dejar zanjadas ciertas cuestiones de su vida? Como normas generales y según lo descrito en la literatura ${ }^{10-15}$ acerca de cómo dar malas noticias:

- Debemos ofrecer información objetiva de todo aquello que el paciente quiera saber y solo lo que quiera saber. Para ello deberemos estar formados en estrategias de comunicación (empatía, escucha activa, manejo de los silencios, etc), dirigidas a aumentar la aceptación y disminuir el miedo del enfermo (counselling) para evitar, en la medida de lo posible tanto el pacto del silencio como el encarnizamiento informativo. Es lo que se ha dado en llamar "la verdad soportable".
- Este proceso será dinámico y bidireccional: la información debe ser efectiva para lo que deberemos saber explorar lo que el paciente ha entendido de aquello que hemos intentado transmitir y las emociones que esta información ha generado en él.

- Se trata de un proceso, no de un hecho puntual: la transmisión se irá haciendo de modo secuencial, según el paciente pueda asimilarlo.

- En el proceso de comunicación no solo están en juego conocimientos técnicos y científicos sino también valores: debemos saber explorar los valores del enfermo para poder ayudarle en la toma de decisiones. Y a la vez se nos pedirá en muchas ocasiones que transmitamos nuestros propios valores: "Doctor, ¿qué haría usted si fuera su padre?."

- En todo este proceso de comunicación tendremos en cuenta que la incertidumbre tanto del profesional como del paciente que recibe malas noticias, con respecto a múltiples aspectos, será una constante: indicación o no indicación de un tratamiento paliativo (ej. ¿me someto a una cirugía con intención paliativa? ¿me compensarán los efectos de una quimioterapia o radioterapia paliativas? ¿podré aguantar todo el proceso sin ser una carga? ¿cuánto tiempo me queda con ello o sin ello?...). Debemos aprender a manejar todo el proceso dentro de unos márgenes aceptables de incertidumbre. Más allá de los elementos racionales deberemos saber estar atentos a los elementos emocionales de toda esta información, vinculados muchas veces a la incertidumbre y otras a los miedos profundos a la muerte que pronto llegará.

\section{Autonomía del paciente y planificación anticipada}

En el modelo de relación sanitaria deliberativo, el paciente es el "protagonista", el sujeto fundamental del proceso y el profesional es el "coprotagonista". Las decisiones se tomarán de manera compartida pero poniendo la autonomía ${ }^{16}$ por delante de la beneficencia. La autonomía del paciente no debe centrarse en la firma de documentos escritos (documentos de Consentimiento informado) para procedimientos y/o tratamientos de especial riesgo. Decisiones como si un paciente quiere o no quimioterapia con intención paliativa, cirugía paliativa, opiáceos, hacerse otro TAC, ponerse una sonda para alimentarse, conectarse a un respirador, desconectarse de él una vez conectado, morir en su domicilio o en el hospital, serán un ejercicio de su autonomía ${ }^{17}$ (Tabla 1).

Para que una decisión sea plenamente autónoma ${ }^{17}$ deben darse las siguientes condiciones: decisión informada, voluntaria, libre y sin coacción, coherente según sus valores y ejercida por un paciente mentalmente competente.

De manera anticipada, en todo este proceso que se inicia desde el momento en que a un paciente se le diagnostica una enfermedad incurable y progresiva se pueden ir planificando decisiones para un futuro en que tal vez el paciente no pueda decidir bien porque su capacidad se pueda ver disminuida 0 porque surjan situaciones urgentes que impidan tomar una decisión pensada y prudente. Los documentos de Volunta- 
Tabla 1. Artículos de la Ley básica reguladora de la autonomía del paciente y de derechos y obligaciones en materia de información y documentación clínica ${ }^{17}$

Artículo 2: Principios básicos.

3. El paciente o usuario tiene derecho a decidir libremente, después de recibir la información adecuada, entre las opciones clínicas disponibles.

4. Todo paciente 0 usuario tiene derecho a negarse al tratamiento, excepto en los casos determinados en la Ley. Su negativa al tratamiento constará por escrito

6. Todo profesional que interviene en la actividad asistencial está obligado no solo a la correcta prestación de sus técnicas, sino a cumplimiento de los deberes de información y documentación clínica, y al respeto de las decisiones adoptadas libre y voluntariamente por el paciente.

Artículo 8: Consentimiento informado.

5. El paciente puede revocar libremente su consentimiento por escrito en cualquier momento.

Artículo 11: Instrucciones previas.

1. Por el documento de instrucciones previas, una persona mayor de edad, capaz y libre, manifiesta anticipadamente su voluntad, con objeto de que ésta se cumpla en el momento en que llegue a situaciones en cuyas circunstancias no sea capaz de expresarlos personalmente, sobre los cuidados y el tratamiento de su salud ...

des Anticipadas o Instrucciones Previas ${ }^{17}$ (en Galicia Registro Gallego de Instrucciones Previas, REGAIP) permiten expresar por escrito durante la capacidad para cuando no sea posible expresar la propia decisión.

Pero sin necesidad de dejar un documento escrito, pequeñas 0 grandes decisiones pueden ir siendo planificadas ${ }^{5,18}$ según los valores del paciente, que el profesional ayudará a explicitar: ej. Llegado el caso de que tenga un sufrimiento intenso por dificultad respiratoria que precise tal vez dormirle para que no sufra, ¿preferiría que le durmiéramos?, ¿prefiere que eso sea en casa o preferiría ir al hospital?, ¿aunque esto suponga que no se despierte? Se puede planificar desde asuntos muy evidentes como los económicos, a quién legar sus pertenencias, cómo le gustaría su funeral, dónde esparcir sus cenizas, etc.

\section{Proceso deliberativo}

La toma de decisiones compartida requiere un proceso informativo efectivo, según lo ya expuesto, permitir el ejercicio de la autonomía del paciente en función de lo que conoce y de sus valores, pero también influirán las opiniones del profesional que, en numerosas ocasiones, serán requeridas por el paciente. Además se intentará conjugar los valores del paciente con los de su familia más cercana y/o cuidador habitual, que, a veces, pueden entrar en conflicto.

La decisión que se toma (las múltiples decisiones que deberán tomarse a lo largo de toda la enfermedad) será en cada momento el resultado de la consideración de los diferentes valores en juego, de los valores en conflicto en caso de que lo haya, y se decidirá el mejor curso de acción, que será el más prudente ${ }^{19}$, el más ponderado, aquel que lesione lo menos posible todos los valores en juego. Las obligaciones del profesional en este aspecto están en, una vez transmitida la información de la mejor manera posible, ayudar a visibilizar todos los valores que hay detrás de cada elección, con sus ventajas e inconvenientes y con sus incertidumbres, para, en función del ejercicio de su autonomía, ayudar al paciente a tomar la mejor decisión.

\section{Acompañamiento}

Este modelo de TDC tiene otro aspecto fundamental, el acompañamiento, que estará presente en todo el proceso y que, junto a la transmisión de la información y la toma de decisiones que le competen, se constituye en un elemento clave. El acompañamiento ${ }^{20}$ es el proceso en el que el profesional se constituye en coprotagonista, decide no mirar a otro lado ni abandonar a su suerte al paciente en este a veces difícil camino, es "el experto técnico" que siempre está ahí, para intentar solucionar aquellos aspectos concretos que tengan solución, para acompañar cuando no se pueda solucionar, accesible, presente, constante, y sabiendo entender el sufrimiento que en muchas ocasiones rodea a todo el proceso: sufrimiento porque se nos cae el pelo, porque adelgazamos, porque nos desfiguramos, porque no comemos, porque perdemos el trabajo, porque estamos hinchados como pelotas, porque podemos perder nuestra relación de pareja, porque nuestros hijos son pequeños, porque se muere nuestro hijo, porque no me quiero morir...

Las actitudes básicas en este proceso son tres ${ }^{20}$.

- Hospitalidad: es la virtud que nos permite romper la estrechez de nuestros miedos y abrir nuestras casas al extraño, es la habilidad para atender al huésped. Se trata de crear un espacio de seguridad y confianza para que el paciente sea él mismo ante nosotros, se sienta aceptado y valorado tal cual es.

- Presencia: es la capacidad para estar en lo que estamos, entregados a la relación que estamos teniendo con el paciente, mostrándole nuestra disponibilidad, sin prisas, "presentes".

- Compasión: es la actitud por la que, desde la conmoción interna ante la necesidad o el sufrimiento de la persona, nos sentimos movidos a una acción lo más eficiente posible para acompañarlo, ayudarlo a afrontar y paliar su sufrimiento. El mensaje que el paciente recibe es: "no estoy solo en este difícil proceso, quien me escucha es una persona como yo, con sus virtudes y debilidades, y realmente está interesado en ayudarme, cree más que yo en mis propias posibilidades para afrontar esta situación".

\section{Caso clínico (continuación)}

Un año después el neumólogo contacta de nuevo con HADO para tratamiento antibiótico endovenoso por infección respiratoria. En esta ocasión observamos al paciente con un deterioro importante con respecto a la movilidad de extremidad superior derecha y, con ello, de la capacidad para comunicarse. El proceso era dificultoso, manejaba el tablero de letras con dificultad, así como el ordenador. En este tiempo había escrito dos libros de poesía y relatos cortos. Parecía triste, tal vez por la pérdida de expresividad facial. 
Nos indica que quiere hablar con nosotros sin que su esposa esté presente. El proceso de comunicación es largo y penoso, lo que genera en él muestras de ansiedad. Manifiesta su inquietud, porque lo ha oído en los medios, acerca de dejar un documento de Instrucciones Previas para "cuando no pueda comunicarse de ninguna manera". Quiere saber lo que es legal y lo que no. Quiere saber si puede solicitar la retirada del respirador y si eso es eutanasia o no. Y también dice que no puede hablar de estos temas con su mujer, que ella lo evita, dice que "eso no es posible y nada más".

La mujer por su parte nos dice que está deprimido, que realiza numerosos intentos de desconexión del respirador, consiguiéndolo en alguno de ellos con la mano derecha, y que en los últimos meses dice que no quiere seguir viviendo así. Hablamos también con la cuidadora que les ayuda, que corrobora ambas versiones, y confirma también que cada vez es más dificultosa la comunicación.

El equipo de HADO considera que se trata de un paciente autónomo, libre para decidir, sin coacción y que solicita información acerca de sus derechos, de cara a planificar su atención futura. En este contexto se asesora al paciente acerca de lo que es limitación del esfuerzo terapéutico, de lo que significa rechazo de tratamiento o no inicio, que ambos supuestos son legales en nuestro país. Explicamos lo que es eutanasia y que esto no es legal en nuestro medio. Clarificamos que lo que él solicita es un rechazo de tratamiento en forma de retirada de medidas ya iniciadas. Nos pide que clarifiquemos todos estos conceptos con su mujer.

Tras este asesoramiento nos indicó que quería otorgar su documento de instrucciones previas y depositarlo en el REGAIP. Pero también nos dijo que, de todos modos, él ya deseaba solicitar que le retirasen las medidas de soporte vital y que por el momento, era capaz. Nos quedaban varias dudas: la mujer se oponía frontalmente a esta decisión. No había motivos de ideología de fondo; no quería perder a su marido. Y también nos quedaba la duda de si el paciente padecía una depresión, dada la insistencia actual en la retirada de la VMI. Se solicitó interconsulta al Servicio de Psiquiatría, quien visitó al paciente en varias ocasiones así como a su esposa. Se ajustó el tratamiento antidepresivo, y asimismo se recomendó a la esposa terapia psicológica de apoyo. También el neumólogo visitó al paciente. En principio se consiguió una respuesta aceptable, desapareciendo por un tiempo los intentos de retirada del respirador. Explicamos nuestra disponibilidad continua, y nuestro compromiso de intentar cumplir su voluntad, según sus valores. Fue alta temporal.

Pasaron 2-3 meses y desde el Servicio de Urgencias contactaron de nuevo con HADO. Se había desconectado del respirador, y cuando su mujer oyó la alarma del ventilador estaba cianótico y sin respuesta. Ella lo volvió a conectar de manera automática y llamó al 061 que lo trasladó al Hospital. Tras varias horas recuperó nivel de conciencia. La mujer nos contó que había llegado a la conclusión de que la petición era cierta pero que ella era incapaz de dejarlo sin conectar si él se desconectaba. Cada vez se podía comunicar peor, generando esto numerosos problemas diarios. Nos contó que estaba intentando hacerse a la idea de que un día su marido moriría y que empezaba a entender que esa era su voluntad.

Nuevamente HADO acudió a su domicilio. Volvimos a contactar con el psiquiatra quien realizó nueva valoración. Según su informe no era cuestión de depresión sino de valores. Vemos al paciente tranquilo, con mirada serena. De nuevo al vernos, con gran dificultad, nos manifiesta que nuestro compromiso con él era CUMPLIR SUS VALORES: "esto no es vida para mí, no es digno para mí, me siento como un muñeco de trapo, al que tienen que limpiar, dar de comer, asistir para respirar; soy una carga. Ya he terminado mi libro, no tengo necesidad de completar nada más, estoy en paz con mi esposa, ahora entiende lo que yo quiero. NO QUIERO SEGUIRVIVIENDO ASI". El equipo y los otros especialistas consultados estaban de acuerdo en que era una petición libre, sin coacción, coherente, en un paciente capaz, sin otra enfermedad intercurrente que pudiera influir, y reiterada en el tiempo. Solicitamos valoración de un facultativo ajeno al servicio a fin de aumentar la objetividad en la decisión y al mismo tiempo de asesorarnos en los aspectos técnicos del procedimiento.

Nos pidieron él y su mujer una reunión con su familia más allegada, con mucha relación e implicación con el paciente, con el fin de aclarar las dudas de cada uno de ellos. Se realizó una tarde, según deseo del paciente. Decidió detalles acerca del final: lugar, compañía, música. . . Le quedaban "algunos asuntos que cerrar".

Un mes después la mujer nos avisó para decirnos que todo estaba listo para proceder a la retirada de medidas de soporte vital.

No decisión about me, without me` (Ninguna decisión para mí, sin mî) A. Coulter, A. Collins

Este trabajo es una revisión de una versión previa, expuesta en la Jornada "Bioética: un instrumento de calidad para la atención sanitaria en el sistema publico de salud de Galicia", celebrada en Santiago de Compostela el 25 de septiembre de 2012 (http://www.sergas.es/cas/Publicaciones/Docs/AtEspecializada/PDF-2237-es.pdf)

\section{Bibliografía}

1. Barbero J, Prados C, González A. Hacia un modelo de toma de decisiones compartida para el final de la vida. Psicooncología 2011; 8: 143-168.

2. President's Commission for the Study of Ethical Problems in Medicine and Biomedical and Behavioral Research. Making Health Care Decisions. The ethical and legal implications of informed consent in the patient-practitioner relationship. U.S. Government Printing Office, Washington, 1982.

3. Charles C, Gafni A, Whelan T. Shared decision-making in the medical encounter: what does it mean? (Or it takes at least two to tango). Soc Sci Med 1997; 44: 651-61.

4. Ruiz-Azarola A, Perestelo-Pérez L. Participación ciudadana en salud: formación y toma de decisiones compartida. Informe SESPAS 2012. Gac Sanit. 2012; 26 (S): 158-161.

5. Os coidados ao final da vida. Documento de recomendacions. Consellería de Sanidade-Servicio Galego de Saúde: Comisión Galega de Bioética. Santiago de Compostela 2012. Disponible en: http://www.sergas.es/Docs/Bioetica/Cuidados\%20 CAST_240912_link.pdf, consultado el 6 de agosto de 2013.

6. Elwyn el all. Shared decision making and the concept of equipoise: the competences of involving patients in healthcare choices. British Journal of General Practice, 2000, 50: 892-897.

7. Coulter A, Collins A. Making Shared Decision-Making a reality: No decision about me, without me. The King's Fund, London 2011.

8. Garrido Sanjuán J.A. Los cuidados al final de la vida: documento de recomendaciones. Una aportación desde la bioética a la calidad de la atención sanitaria en Galicia. Galicia clínica 2013; 74 (2): 51-57.

9. Gracia D. Los cambios en la relación médico-enfermo. Med Clin (Barc) 1989; 93: 100-2.

10. Buckman R. How to break bad news. A guide for health care professionals. Baltimore: Ed. John Hopkins, 1992.

11. Coulter A. Patient information and shared decision-making in cancer care. British Journal of Cancer, 2003; 89: S15-S16.

12. Barbero J. El derecho del paciente a la información: el arte de comunicar. An Sist Sanit Navar 2006; 29: 19-27.

13. Borrell, F. Comunicación y salud. Toma de decisiones en una práctica clínica centrada en el paciente, en: Ciclo de Conferencias "Medicina y Humanidades", Real Academia Nacional de Medicina, Madrid, 14 de octubre de 2010.

14. Lizarraga S. Ayarra M. Cabodevila I. La comunicación como piedra angular de la atención al paciente oncológico avanzado. Bases para mejorar nuestras habilidades. Aten Primaria, 2006; 38 (S2): 7-13.

15. Novo JM, Martínez FJ. Doce más una estrategias erróneas para dar malas noticias. Galicia Clínica 2012; 73: 117-119.

16. Simón P. El consentimiento informado: alianza y contrato, deliberación y decisión. En: Couceiro A (ed.). Ética en Cuidados Paliativos. Ed. Triacastela, Madrid, 2004.

17. Ley $41 / 2002$, de 14 de noviembre, básica reguladora de la autonomía del paciente y de derechos y obligaciones en materia de información y documentación clínica. BOE (n² 274), de 15 de noviembre de 2002, páginas 40126-40132.

18. Simón P, Tamayo M.I, Esteban M. Planificación Anticipada de las Decisiones. Guía de apoyo para profesionales. Junta de Andalucía. Consejería de salud y bienestar social. Sevilla, 2013. Disponible en: http://www.juntadeandalucia.es/salud/sites/csalud/contenidos/Informacion_General/c_2_c_8_voluntad_vital_anticipada/planificacion_anticipada_de_decisiones_SSPA.pdf, consultado el 6 de agosto de 2013.

19. Gracia D. Teoría y práctica de la deliberación moral. En: Bioética: el estado de la cuestión. Triacastela, Madrid, 2011.

20. Benito E, Maté J, Pascual A. Estrategias para la detección, exploración y atención del sufrimiento en el paciente. FMC 2011; 18: 392-400. 